H. HIRAMATU

KODAI MATH. J.

3 (1980), 397-406

\title{
RIEMANNIAN MANIFOLDS ADMITTING A PROJECTIVE VECTOR FIELD
}

\author{
By Hitosi HiRAmatu
}

\section{$\S 1$. Introduction.}

Let $M$ be a connected Riemannian manifold of dimension $n$ covered by a system of coordinate neighborhoods $\left\{U ; x^{h}\right\}$, where, here and in the sequel, the indices $h, i, j, k, \cdots$ run over the range $\{1,2, \cdots, n\}$ and let $g_{j i},\left\{{ }_{j}{ }_{i}{ }_{i}\right\}, \nabla_{\jmath}, K_{k j i}{ }^{h}$, $K_{j i}$ and $K$ be respectively the metric tensor, the Christoffel symbols formed with $g_{j i}$, the operator of covariant differentiation with respect to $\left\{{ }_{j}{ }_{i}{ }_{i}\right\}$, the curvature tensor, the Ricci tensor and the scalar curvature of $M$.

A vector field $v^{h}$ on $M$ is called a projective vector field if it satisfies

$$
L_{v}\left\{{ }_{j}{ }_{i}\right\}=\nabla_{j} \nabla_{\imath} v^{h}+v^{k} K_{k j i}{ }^{h}=\delta_{j}^{h} \rho_{i}+\delta_{\imath}^{h} \rho_{\jmath}
$$

for a certain covariant vector field $\rho_{\imath}$, called the associated covariant vector field of $v^{h}$, where $L_{v}$ denotes the operator of Lie derivation with respect to the vector field $v^{h}$. In particular, if $\rho_{\imath}$ in (1.1) is zero vector field then the projective vector field $v^{h}$ is called an affine vector field. When we refer in the sequel to a projective vector field $v^{h}$, we always mean by $\rho_{\imath}$ the associated covariant vector field appearing in (1.1).

Recently, the present author $[1,2]$ proved a series of integral inequalities in a compact and orientable Riemannian manifold with constant scalar curvature admitting a projective vector field and then obtained necessary and sufficient conditions for such a Riemannian manifold to be isometric to a sphere.

The purpose of the present paper is to continue the work of the present author $[1,2]$ and to prove the following theorem.

THEOREM A. If a connected, compact, orientable and simply connected Riemannian manifold $M$ with constant scalar curvature $K$ of dimension $n>1$ admits a non-affine projective vector field $v^{h}$, then $M$ is globally isometric to a sphere of radius $\sqrt{n(n-1) / K}$ in the Euclidean $(n+1)$-space.

In the sequel, we need the following theorem due to Obata [3]. (See also [4].)

THEOREM B. Let $M$ be a complete, connected and simply connected Rieman-

Received July 25, 1979 
nian manıfold. In order for $M$ to admit a non-trivial solution $\varphi$ of a system of partial differential equations

$$
\nabla_{j} \nabla_{i} \varphi_{h}+k\left(2 \varphi_{j} g_{i h}+\varphi_{i} g_{j h}+\varphi_{h} g_{j i}\right)=0,
$$

where $\varphi_{h}=\nabla_{h} \varphi, k$ being a positive constant, it is necessary and sufficient that $M$ is globally isometric to a sphere of radius $1 / \sqrt{k}$ in the Euclidean $(n+1)$-space.

We assume in this paper that Riemannian manifolds under consideration are connected.

\section{$\S 2$. Preliminaries.}

We consider a projective vector field $v^{h}$ on a Riemannian manifold $M$ of dimension $n$. From (1.1), we have easily

$$
\nabla_{j} \nabla_{t} v^{t}=(n+1) \rho_{j}
$$

and

$$
\nabla^{j} \nabla_{\jmath} v^{2}+K_{\jmath}{ }^{2} v^{\jmath}=2 \rho^{\imath},
$$

where $\nabla^{\jmath}=g^{j i} \nabla_{\imath}$ and $\rho^{\imath}=g^{i \hbar} \rho_{h}$. (2.1) shows that the associated covariant vector field $\rho_{\rho}$ is gradient. Putting

$$
\rho=\frac{1}{n+1} \nabla_{t} v^{t}
$$

we have

$$
\rho_{j}=\nabla_{j} \rho
$$

We have, from (1.1),

$$
\nabla_{\jmath}\left(\nabla_{\imath} v_{h}+\nabla_{h} v_{\imath}\right)=2 \rho_{j} g_{\imath h}+\rho_{\imath} g_{\jmath h}+\rho_{h} g_{\jmath \imath},
$$

from which

$$
\nabla, L_{v} g^{i n}=-2 \rho_{j} g^{i h}-\rho^{i} \delta_{\jmath}^{h}-\rho^{h} \delta_{\jmath}^{2}
$$

and

$$
\nabla^{j} L_{v} g^{i h}=-2 \rho^{j} g^{2 h}-\rho^{2} g^{j h}-\rho^{h} g^{j i},
$$

where $v_{h}=v^{2} g_{i n}$.

Substituting (1.1) into the formula [5]

we find

$$
L_{v} K_{k j i}{ }^{h}=\nabla_{k} L_{v}\left\{{ }_{j}{ }^{h}\right\}-\nabla_{j} L_{v}\left\{{ }_{k}{ }_{i}{ }_{i}\right\},
$$




$$
L_{v} K_{k j i}^{h}=-\delta_{k}^{h} \nabla_{j} \rho_{i}+\delta_{j}^{h} \nabla_{k} \rho_{i},
$$

from which

$$
L_{v} K_{j i}=-(n-1) \nabla_{j} \rho_{\imath} .
$$

We define a tensor field $G_{j i}[1,2]$ on $M$ by

$$
G_{j i}=K_{j i}-\frac{K}{n} g_{j i},
$$

which satisfies

$$
G_{\jmath i}=G_{\imath \jmath}, \quad G_{j i} g^{j i}=0 .
$$

If $G_{j i}=0$ for $n>2$ then $M$ is an Einstein manifold and $K$ is a constant.

The projective curvature tensor field $P_{k j i}{ }^{h}$ on $M$ is defined by

$$
P_{k j i}{ }^{h}=K_{k \jmath i}{ }^{h}-\frac{1}{n-1}\left(\delta_{k}^{h} K_{j i}-\delta_{\jmath}^{h} K_{k \imath}\right) .
$$

The tensor field $P_{k j i}^{h}$ satisfies

$$
\begin{gathered}
P_{k j i}{ }^{h}=-P_{\jmath k \imath}{ }^{h}, \\
P_{k j i}{ }^{h}+P_{i k j}{ }^{h}+P_{\jmath i k}{ }^{h}=0, \\
P_{t j i}{ }^{t}=0, \quad P_{k \jmath t}{ }^{t}=0
\end{gathered}
$$

and

$$
P_{k j i n} g^{j i}=\frac{n}{n-1} G_{k h},
$$

where $P_{k j i h}=P_{k j i}{ }^{t} g_{t h}$.

It is known that if $n=2$ then $P_{k j l}{ }^{h}=0$ and if $P_{k j i}{ }^{h}=0$ for $n>2$ then $M$ is projectively flat.

If the scalar curvature $K$ is a constant, then, using

$$
\nabla^{j} K_{j \imath}=\frac{1}{2} \nabla_{i} K=0
$$

and

$$
\nabla_{t} K_{k j i}{ }^{t}=\nabla_{k} K_{j i}-\nabla_{\jmath} K_{k \imath},
$$

which can be obtained from the Bianchi identity

we have

$$
\nabla_{l} K_{k j i}{ }^{n}+\nabla_{j} K_{l k \imath}{ }^{n}+\nabla_{k} K_{j l \imath}{ }^{h}=0,
$$

$$
\nabla^{k} P_{k \jmath i}{ }^{h}=\frac{n-2}{n-1} \nabla^{h} G_{\jmath \imath}-\nabla_{i} G_{\jmath}{ }^{h}
$$


where $G_{j}{ }^{h}=G_{j i} g^{i h}$.

For a projective vector field $v^{h}$ on a Riemannian manifold $M$, we have, for the tensor field $G_{j i}$,

$$
L_{v} G_{j i}=-\nabla_{j} w_{i}-\nabla_{i} w_{j}
$$

if the scalar curvature $K$ is a constant, where we have put

$$
w^{h}=\frac{n-1}{2} \rho^{n}+\frac{K}{n} v^{n}
$$

and $w_{\imath}=g_{i \hbar} w^{h}$, and, for the projective curvature tensor field $P_{k j \imath}{ }^{h}$,

$$
L_{v} P_{k j 2}{ }^{n}=0 .
$$

\section{§3. Proof of Theorem A.}

In this section, we prove Theorem A. For this purpose, we need a series of lemmas.

LEMMA 1. In a compact and orentable Riemannan manifold $M$, we have

$$
\int_{M}\left(\nabla_{\imath} f\right)\left(\nabla^{i} h\right) d V=-\int_{M} f \Delta h d V=-\int_{M} h \Delta f d V
$$

for any functions $f$ and $h$ on $M$, where $\Delta=g^{j i} \nabla_{j} \nabla_{\imath}$ and $d V$ denotes the volume element of $M$.

Proof. This follows from

$$
0=\int_{M} \nabla_{i}\left(f \nabla^{\imath} h\right) d V=\int_{M}\left(\nabla_{\imath} f\right)\left(\nabla^{\imath} h\right) d V+\int_{M} f \Delta h d V
$$

and

$$
0=\int_{M} \nabla_{i}\left(h \nabla^{i} f\right) d V=\int_{M}\left(\nabla_{i} h\right)\left(\nabla^{\imath} f\right) d V+\int_{M} h \nabla f d V .
$$

LEMMA 2. If, in a compact and orientable Rremannian manifold $M$, a nonconstant function $\varphi$ satisfies a system of partial differential equations

$$
\nabla_{j} \nabla_{i} \varphi_{h}+k\left(2 \varphi_{j} g_{i h}+\varphi_{i} g_{j h}+\varphi_{h} g_{j i}\right)=0,
$$

where $\varphi_{h}=\nabla_{h} \varphi$, $k$ being a constant, then the constant $k$ is necessarily positive.

Proof. Transvecting (3.2) with $g^{i n}$, we have

from which and Lemma 1,

$$
\nabla_{j} \Delta \varphi+2(n+1) k \varphi_{j}=0
$$




$$
\begin{aligned}
k \int_{M} \varphi_{j} \varphi^{\jmath} d V & =-\frac{1}{2(n+1)} \int_{M}\left(\nabla_{j} \Delta \varphi\right) \varphi^{\jmath} d V \\
& =\frac{1}{2(n+1)} \int_{M}(\Delta \varphi)^{2} d V
\end{aligned}
$$

where $\varphi^{j}=g^{j i} \varphi_{i}$. Since $\varphi$ is a non-constant function, two integral inequalities

$$
\int_{M} \varphi_{j} \varphi^{j} d V>0, \quad \int_{M}(\Delta \varphi)^{2} d V>0
$$

hold and consequently $k$ must be positive.

LEMMA 3. If a complete and simply connected Riemannian manifold $M$ with positive constant scalar curvature $K$ of dimension $n>1$ admits a non-affine projective vector fleld $v^{h}$ and if the vector field $w^{h}$ defined by (2.19) is a Killing vector field, then $M$ is globally isometric to a sphere of radius $\sqrt{n(n-1) / K}$ in the Euclidean $(n+1)$-space.

Proof. We have, from (1.1),

$$
\nabla_{j}\left(\nabla_{\imath} v_{h}+\nabla_{h} v_{\imath}\right)=2 \rho_{j} g_{i h}+\rho_{i} g_{j h}+\rho_{h} g_{j i} .
$$

Since $w^{h}$ is a Killing vector field, we have

or, equivalently,

$$
\nabla_{i} w_{h}+\nabla_{h} w_{i}=0
$$

$$
(n-1) \nabla_{i} \rho_{h}+\frac{K}{n}\left(\nabla_{\imath} v_{h}+\nabla_{h} v_{\imath}\right)=0,
$$

from which and the above relation, we find

$$
\nabla_{j} \nabla_{i} \rho_{h}+\frac{K}{n(n-1)}\left(2 \rho_{j} g_{i h}+\rho_{i} g_{j h}+\rho_{h} g_{j i}\right)=0,
$$

and consequently the lemma follows from Theorem B.

Remark. Using Lemma 2, we see that if, in Lemma 3, $M$ is compact and orientable then we can remove the positiveness of the scalar curvature $K$.

LEMMA 4. For a projective vector field $v^{h}$ on a compact and orientable Riemannian manifold $M$ with constant scalar curvature $K$ of dimension $n>1$, we have

$$
\begin{aligned}
\int_{M} G_{j i} \rho^{j} w^{2} d V= & \frac{2}{n-1} \int_{M}\left(\Delta_{t} w^{t}\right)^{2} d V \\
& -\frac{1}{2(n-1)} \int_{M}\left(\nabla_{j} w_{i}+\nabla_{i} w_{j}\right)\left(\nabla^{\jmath} w^{2}+\nabla^{i} w^{j}\right) d V .
\end{aligned}
$$


Proof. By using an identity

we have

$$
\nabla^{j} \nabla_{j} \rho_{i}-K_{j i} \rho^{j}-\nabla_{i} \Delta \rho=0,
$$

$$
\int_{M} G_{j i} \rho^{j} w^{2} d V=-\int_{M}\left(\nabla_{i} \Delta \rho\right) w^{2} d V+\int_{M}\left(\nabla^{j} \nabla_{j} \rho_{\imath}\right) w^{2} d V-\frac{K}{n} \int_{M} \rho_{i} w^{2} d V .
$$

Here we notice that we have, using (2.1) and (2.19),

$$
\begin{aligned}
& -\int_{M}\left(\nabla_{i} \Delta \rho\right) w^{2} d V=\int_{M}(\Delta \rho) \nabla_{i} w^{2} d V \\
& =\int_{M}\left[\nabla_{t}\left\{\frac{2}{n-1} w^{t}-\frac{2 K}{n(n-1)} v^{t}\right\}\right] \nabla_{i} w^{2} d V \\
& =\frac{2}{n-1} \int_{M}\left(\nabla_{t} w^{t}\right)^{2} d V-\frac{2 K}{n(n-1)} \int_{M}\left(\nabla_{t} v^{t}\right) \nabla_{i} w^{2} d V \\
& =\frac{2}{n-1} \int_{M}\left(\nabla_{t} w^{t}\right)^{2} d V+\frac{2 K}{n(n-1)} \int_{M}\left(\nabla_{i} \nabla_{t} v^{t}\right) w^{2} d V \\
& =\frac{2}{n-1} \int_{M}\left(\nabla_{t} w^{t}\right)^{2} d V+\frac{2(n+1) K}{n(n-1)} \int_{M} \rho_{i} w^{2} d V .
\end{aligned}
$$

Consequently, we have

$$
\begin{aligned}
& \int_{M} G_{j i} \rho^{\jmath} w^{2} d V \\
& \quad=\frac{2}{n-1} \int_{M}\left(\nabla_{t} w^{t}\right)^{2} d V+\int_{M}\left(\nabla^{j} \nabla_{j} \rho_{i}\right) w^{2} d V+\frac{(n+3) K}{n(n-1)} \int_{M} \rho_{i} w^{2} d V,
\end{aligned}
$$

from which, using

$$
\nabla^{\jmath}\left(\nabla_{j} v_{i}+\nabla_{\imath} v_{j}\right)=\nabla^{j} L_{v} g_{j i}=(n+3) \rho_{i}
$$

$$
\begin{aligned}
\int_{M} G_{j i} \rho^{j} w^{2} d V & =\frac{2}{n-1} \int_{M}\left(\nabla_{t} w^{t}\right)^{2} d V \\
& +\frac{1}{n-1} \int_{M}\left[\nabla^{j}\left\{\nabla_{j}\left(\frac{n-1}{2} \rho_{i}+\frac{K}{n} v_{\imath}\right)+\nabla_{i}\left(\frac{n-1}{2} \rho_{j}+\frac{K}{n} v_{j}\right)\right\}\right] w^{2} d V \\
= & \frac{2}{n-1} \int_{M}\left(\nabla_{t} w^{t}\right)^{2} d V+\frac{1}{n-1} \int_{M}\left\{\nabla^{j}\left(\nabla_{j} w_{i}+\nabla_{i} w_{j}\right)\right\} w^{2} d V \\
= & \frac{2}{n-1} \int_{M}\left(\nabla_{t} w^{t}\right)^{2} d V-\frac{1}{n-1} \int_{M}\left(\nabla_{j} w_{i}+\nabla_{i} w_{j}\right) \nabla^{\jmath} w^{2} d V \\
= & \frac{2}{n-1} \int_{M}\left(\nabla_{t} w^{t}\right)^{2} d V-\frac{1}{2(n-1)} \int_{M}\left(\nabla_{j} w_{i}+\nabla_{i} w_{j}\right)\left(\nabla^{j} w^{2}+\nabla^{i} w^{j}\right) d V
\end{aligned}
$$


LEMMA 5. For a projective vector field $v^{h}$ on a compact and orientable Riemannian manifold $M$ with constant scalar curvature $K$, we have

$$
\int_{M}\left(\nabla^{j} L_{v} G_{j i}\right) w^{\imath} d V=\frac{1}{2} \int_{M}\left(\nabla_{j} w_{i}+\nabla_{i} w_{j}\right)\left(\nabla^{\jmath} w^{\imath}+\nabla^{\imath} w^{j}\right) d V
$$

Proof. Integrating

$$
\begin{aligned}
& \nabla^{\jmath}\left\{\left(\nabla_{j} w_{i}+\nabla_{i} w_{j}\right) w^{i}\right\} \\
& \quad=\left\{\nabla^{j}\left(\nabla_{j} w_{i}+\nabla_{i} w_{j}\right)\right\} w^{2}+\left(\nabla_{j} w_{i}+\nabla_{i} w_{j}\right) \nabla^{\jmath} w^{\imath} \\
& \quad=\left\{\nabla^{j}\left(\nabla_{j} w_{i}+\nabla_{i} w_{j}\right)\right\} w^{2}+\frac{1}{2}\left(\nabla_{j} w_{i}+\nabla_{i} w_{j}\right)\left(\nabla^{\jmath} w^{2}+\nabla^{\imath} w^{j}\right)
\end{aligned}
$$

over $M$ and using (2.18), we have (3.4).

LEMMA 6. For a projective vector field $v^{h}$ on a compact and orientable Riemannian manifold $M$ with constant scalar curvature $K$ of dimension $n>1$, we have

$$
\begin{aligned}
\int_{M}\left(\nabla^{j} L_{v} G_{j}{ }^{i}\right) w_{i} d V= & -\frac{6}{n-1} \int_{M}\left(\nabla_{t} w^{t}\right)^{2} d V \\
& +\frac{n+2}{2(n-1)} \int_{M}\left(\nabla_{j} w_{i}+\nabla_{i} w_{j}\right)\left(\nabla^{j} w^{i}+\nabla^{i} w^{j}\right) d V
\end{aligned}
$$

Proof. Using

$$
\nabla^{\jmath} G_{j i}=\frac{n-2}{2 n} \nabla_{i} K=0
$$

we have

$$
\begin{aligned}
\left(\nabla^{j} L_{v} G_{\jmath}{ }^{i}\right) w_{\imath} & =\left\{\nabla^{j} L_{v}\left(G_{\jmath t} g^{t i}\right)\right\} w_{\imath} \\
& =\left(\nabla^{j} L_{v} G_{j i}\right) w^{\imath}+G_{j t}\left(\nabla^{j} L_{v} g^{t i}\right) w_{\imath} .
\end{aligned}
$$

Substituting (2.7) into this, we find

$$
\left(\nabla^{j} L_{v} G_{j}{ }^{i}\right) w_{i}=\left(\nabla^{j} L_{v} G_{j i}\right) w^{\imath}-3 G_{j i} \rho^{j} w^{\imath} .
$$

Integrating this over $M$ and using Lemmas 4 and 5, we have (3.5).

LEMMA 7. For a projective vector field $v^{h}$ on a campact and orientable Riemannian manifold $M$ with constant scalar curvature $K$ of dimension $n>1$, we have

$$
\begin{aligned}
\int_{M} g^{k j}\left(L_{v} \nabla_{k} G_{j i}\right) w^{i} d V= & -\frac{6}{n-1} \int_{M}\left(\nabla_{t} w^{t}\right)^{2} d V \\
& +\frac{n+2}{2(n-1)} \int_{M}\left(\nabla_{j} w_{i}+\nabla_{i} w_{j}\right)\left(\nabla^{j} w^{i}+\nabla^{\imath} w^{j}\right) d V
\end{aligned}
$$


Proof. We have

$$
\begin{aligned}
g^{k j}\left(L_{v} \nabla_{k} G_{j i}\right) w^{\imath} & =\left(\nabla^{j} L_{v} G_{j i}\right) w^{2}-g^{k j}\left(L_{v}\left\{{ }_{k}{ }_{j}{ }_{j}\right\}\right) G_{t i} w^{2}-g^{k j}\left(L_{v}\left\{{ }_{k}{ }_{k}{ }_{i}\right\}\right) G_{j t} w^{2} \\
& =\left(\nabla^{j} L_{v} G_{j i}\right) w^{2}-3 G_{j i} \rho^{j} w^{2} .
\end{aligned}
$$

Integrating this over $M$ and using Lemmas 4 and 5, we have (3.6).

Now we prove Theorem A. By using

$$
\left(L_{v} P_{k j i}{ }^{h}\right) g^{j i}=\frac{n}{n-1} L_{v} G_{k}{ }^{n}-P_{k j i}{ }^{n} L_{v} g^{j i},
$$

we have

$$
\begin{aligned}
& \left(\nabla^{k} L_{v} P_{k j i}{ }^{h}\right) g^{j i} w_{h}=\frac{n}{n-1}\left(\nabla^{k} L_{v} G_{k}{ }^{h}\right) w_{h} \\
& \quad-\left(\nabla^{k} P_{k j i}{ }^{h}\right)\left(L_{v} g^{j i}\right) w_{h}-P_{k j i}{ }^{h}\left(\nabla^{k} L_{v} g^{j i}\right) w_{h} .
\end{aligned}
$$

Substituting (2.7) and (2.17) into this, we find

$$
\begin{aligned}
& \left(\nabla^{k} L_{v} P_{k j i}{ }^{h}\right) g^{j i} w_{h}=\frac{n}{n-1}\left(\nabla^{k} L_{v} G_{k}{ }^{h}\right) w_{h} \\
& \quad-\frac{n-2}{n-1}\left(\nabla^{h} G_{j i}\right)\left(L_{v} g^{j i}\right) w_{h}+\left(\nabla_{i} G_{j}{ }^{h}\right)\left(L_{v} g^{j i}\right) w_{h}+\frac{n}{n-1} G_{j i} \rho^{j} w^{\imath},
\end{aligned}
$$

from which, integrating over $M$,

$$
\begin{aligned}
& \int_{M}\left(\nabla^{k} L_{v} P_{k j i}{ }^{h}\right) g^{j i} w_{h} d V \\
& =\frac{n}{n-1} \int_{M}\left(\nabla^{j} L_{v} G_{\jmath}{ }^{i}\right) w_{i} d V-\frac{n-2}{n-1} \int_{M}\left(\nabla_{t} G_{j i}\right)\left(L_{v} g^{j i}\right) w^{t} d V \\
& \quad+\int_{M}\left(\nabla_{k} G_{j i}\right)\left(L_{v} g^{k j}\right) w^{2} d V+\frac{n}{n-1} \int_{M} G_{j i} \rho^{j} w^{2} d V .
\end{aligned}
$$

Here we notice that we have, using (2.6) and (2.18),

$$
\begin{aligned}
& -\frac{n-2}{n-1} \int_{M}\left(\nabla_{t} G_{j i}\right)\left(L_{v} g^{j i}\right) w^{t} d V \\
& =\frac{n-2}{n-1} \int_{M} G_{j i}\left(\nabla_{t} L_{v} g^{j i}\right) w^{t} d V+\frac{n-2}{n-1} \int_{M} G_{j i}\left(L_{v} g^{j i}\right) V_{t} w^{t} d V \\
& =-\frac{2(n-2)}{n-1} \int_{M} G_{j i} \rho^{j} w^{\imath} d V-\frac{n-2}{n-1} \int_{M}\left(L_{v} G_{j i}\right) g^{j i} \nabla_{t} w^{t} d V \\
& =-\frac{2(n-2)}{n-1} \int_{M} G_{j i} \rho^{j} w^{\imath} d V+\frac{2(n-2)}{n-1} \int_{M}\left(\nabla_{t} w^{t}\right)^{2} d V
\end{aligned}
$$


and, using

$$
\begin{gathered}
\left(\nabla_{k} G_{j i}\right) g^{k J}=\frac{n-2}{2 n} \nabla_{i} K=0, \\
\int_{M}\left(\nabla_{k} G_{j i}\right)\left(L_{v} g^{k j}\right) w^{2} d V=-\int_{M} g^{k J}\left(L_{v} \nabla_{k} G_{j i}\right) w^{2} d V .
\end{gathered}
$$

Consequently, we have

$$
\begin{aligned}
& \int_{M}\left(\nabla^{k} L_{v} P_{k j i}{ }^{h}\right) g^{j i} w_{h} d V \\
& =\frac{2(n-2)}{n-1} \int_{M}\left(\nabla_{t} w^{t}\right)^{2} d V-\frac{n-4}{n-1} \int_{M} G_{j i} \rho^{j} w^{2} d V \\
& \quad+\frac{n}{n-1} \int_{M}\left(\nabla^{j} L_{v} G_{\jmath}{ }^{i}\right) w_{i} d V-\int_{M} g^{k \jmath}\left(L_{v} \nabla_{k} G_{j i}\right) w^{2} d V,
\end{aligned}
$$

from which, using Lemmas 4, 6 and 7, we have

$$
\begin{aligned}
\int_{M}\left(\nabla^{k} L_{v} P_{k j i}{ }^{h}\right) g^{j i} w_{h} d V= & \frac{2(n-3)}{n-1} \int_{M}\left(\nabla_{t} w^{t}\right)^{2} d V \\
& +\frac{1}{n-1} \int_{M}\left(\nabla_{j} w_{i}+\nabla_{i} w_{j}\right)\left(\nabla^{\jmath} w^{2}+\nabla^{\imath} w^{j}\right) d V .
\end{aligned}
$$

From this and (2.20), we have

$$
\frac{2(n-3)}{n-1} \int_{M}\left(\nabla_{t} w^{t}\right)^{2} d V+\frac{1}{n-1} \int_{M}\left(\nabla_{j} w_{i}+\nabla_{i} w_{j}\right)\left(\nabla^{\jmath} w^{2}+\nabla^{\imath} w^{j}\right) d V=0 .
$$

If $n>2$ then we have, from the above relation,

$$
\int_{M}\left(\nabla_{j} w_{i}+\nabla_{i} w_{j}\right)\left(\nabla^{\jmath} w^{i}+\nabla^{i} w^{j}\right) d V=0
$$

from which

$$
\nabla_{j} w_{i}+\nabla_{i} w_{j}=0
$$

that is, the vector field $w^{h}$ defined by (2.19) is a Killing vector field and Theorem A follows from Lemma 3. If $n=2$ then we have $P_{k j i}{ }^{h}=0$ and hence, from (2.16), $G_{j i}=0$. Consequently, using Lemma 5 , we have

$$
\int_{M}\left(\nabla_{j} w_{i}+\nabla_{i} w_{j}\right)\left(\nabla^{\jmath} w^{2}+\nabla^{\imath} w^{j}\right) d V=0
$$

from which

$$
\nabla_{j} w_{\imath}+\nabla_{i} w_{\jmath}=0
$$

and Theorem A follows from Lemma 3. 


\section{BIBLIOGRAPHY}

[1] H. Hiramatu, On integral inequalities and their applications in Riemannian manifolds, Geometriae Dedicata, 7 (1978), 375-386.

[2] H. Hiramatu, Integral inequalities and their applications in Riemannian manifolds admitting a projective vector field, to appear in Geometriae Dedicata.

[3] M. OBAtA, Riemannian manifolds admitting a solution of a certain system of differential equations, Proc. U.S.-Japan Seminar in Differential Geometry, Kyoto, Japan, 1965, 101-114.

[4] S. Tanno, Some differential equations on Riemannian manifolds, J. Math. Soc. Japan, 30 (1978), 509-531.

[5] K. YANO, Integral formulas in Riemannian geometry, Marcel Dekker, New York, 1970.

Department of Mathematics

FACULTY OF ENGINEERING

KuMAMOTO UNIVERSITY,

KUMAMOTO 860, JAPAN 